Review Article

\title{
Evaluation of Efficacy and Safety of Dan'e-Fukang Soft Extract in the Treatment of Endometriosis: A Meta-Analysis of 39 Randomized Controlled Trials Enrolling 5442 Patients
}

\author{
Yantao Li, ${ }^{1} \mathrm{Te} \mathrm{Li}^{2}$, and Shilin Song ${ }^{3}$ \\ ${ }^{1}$ Department of Prevention, Nankai Hospital, Tianjin Academy of Integrative Medicine, Tianjin 300100, China \\ ${ }^{2}$ Department of Chinese Medicine, Tianjin Hearing Impairment Specialist Hospital, Tianjin 300150, China \\ ${ }^{3}$ Laboratory of Anatomy, School of Integrative Medicine, Tianjin University of Traditional Chinese Medicine, Tianjin 300193, China \\ Correspondence should be addressed to Shilin Song; haiguagua@163.com
}

Received 19 August 2016; Accepted 20 December 2016; Published 27 February 2017

Academic Editor: Shan-Yu Su

Copyright (C) 2017 Yantao Li et al. This is an open access article distributed under the Creative Commons Attribution License, which permits unrestricted use, distribution, and reproduction in any medium, provided the original work is properly cited.

Objective. To systematically evaluate the efficacy and safety of Dan'e-fukang soft extract in endometriosis treatment. Method. PubMed, CNKI, Wanfang Database, VIP, SinoMed, and Cochrane Library were searched. Randomized controlled trials (RCTs) comparing the efficacy of Dan'e-fukang soft extract and conventional western medicines for endometriosis treatment were included. The data were extracted independently by two people and analyzed using RevMan 5.2.0 software. The relative risk (RR) and mean difference (MD) with 95\% confidence intervals were considered as effective outcome indicators. Results. Thirty-nine papers including 5442 patients with endometriosis were included in this study. A meta-analysis revealed that Dan'e-fukang soft extract was more efficient than gestrinone in the treatment of endometriosis ( RR $=1.08,95 \% \mathrm{CI}=1.03$ to $1.15, I^{2}=71 \%, \mathrm{REM}, 18$ trials) and its efficacy was comparable to that of danazol and mifepristone. Dan'e-fukang soft extract was also as effective as gestrinone and mifepristone in terms of relapse rate and relieving dysmenorrhea. The incidence of adverse reactions was lower than that of conventional western medicines. Conclusions. The results of this study showed that Dane-fukang soft extract offers certain advantages in endometriosis treatment, but rigorously designed, strictly implemented RCTs are needed to further validate its efficacy.

\section{Introduction}

Endometriosis (EMs) is an estrogen-dependent gynecological disease in which endometrium-like tissues grows in abnormal sites other than the endometrium, and it can lead to infertility and dysmenorrhea [1]. The global prevalence of endometriosis in women of childbearing age is about $5 \%-15 \%$ [2]. Ectopic lesions at various positions, most commonly in the ovary, and extensive adhesion of pelvic tissues can occur. Laparoscopic surgery is the first choice in the treatment of endometriosis. However, endometriosis treatment without total excision may cause hyperplasia, which leads to recurrence, and the recurrence rate is up to $40 \%$ [3]. The drugs currently used in western medicine include danazol, gestrinone, and mifepristone. However, these drugs have serious side effects, and therefore, there is an urgent need to find new, safe, and effective drugs to treat this disease.

According to traditional Chinese medicine (TCM), the basic pathogenesis of endometriosis is blood stasis. Therefore, the primary therapy is promoting blood circulation to remove blood stasis. Dan'e-fukang soft extract is the first pure TCM drug that received national drug approval number for the treatment of endometriosis. It consists of Salvia, Rhizoma Curcumae, Rhizoma Sparganii, bupleurum root, angelica, liquorice, Rhizoma Corydalis, Radix Paeoniae Rubra, and Rhizoma Cyperi. Most of the constituents in the prescription disseminate into the hepatic circulation and is involved in coordinating qi and blood, promoting blood circulation to remove blood stasis, and relieving pain. 
Animal experiments have shown that Dan'e-fukang soft extract helps in modulating immune functions by enhancing cellular immunity and reducing humoral immunity [4]. Currently, Dan'e-fukang soft extract is widely used in clinical practice in China, but it lacks effective evidencebased support. This study will systemically evaluate the clinical efficacy and safety of Dan'e-fukang soft extract in the treatment of endometriosis to provide a reference for clinical management.

\section{Methods}

\subsection{Inclusion Criteria}

Inclusion Criteria. (1) Type of study is as follows: randomized controlled trial (RCT), either blinding or using placebo, and languages limited to Chinese and English. (2) Study candidates are patients who received a diagnosis of endometriosis; endometriosis sites and patients receiving surgeries are not limited. (3) Interventions are as follows: Dan'e-fukang soft extract treatment alone in the test group; medication doses and treatment are not limited; the control group may include patients without treatment or patients receiving placebo or conventional western medicines. The courses of treatment, methods, and dosage are not limited. (4) Outcome measures are as follows: efficacy rate, recurrence rate, remission rate of dysmenorrhea, CA125, and safety.

2.2. Search Strategies. PubMed, Cochrane Library, VIP, CNKI, Wanfang Database were searched by computer. The search period ranged from their date of foundation to July 1, 2016. The search terms included Dan'e-fukang soft extract (dan e fu kang jian gao), endometriosis, and random. The references to included literatures were also searched.

2.3. Literature Screening. The titles and abstracts of the searched papers were studied by two independent investigators. Trials not obviously meeting the inclusion criteria were excluded. For trials that possibly met the inclusion criteria, the entire papers were further studied to determine if they actually met the inclusion criteria, and they were crosschecked. Disagreements on whether certain trials should be included were resolved through discussions.

\subsection{Methodological Quality Assessments for Included Studies.} Methods recommended by the Cochrane Collaboration were adopted in the methodological quality assessment for RCT, which included six risks of bias assessments: random allocation sequence generation, allocation concealment, blinding, data integrity, selective outcome reporting, and other biases [5]. Entries were considered to have a low risk of bias (low) if they met the criteria and a high risk of bias (high) if they did not meet the criteria. In addition, entries were considered unclear when the paper did not provide enough information for judgment. Methodological quality assessment of clinical trials was performed by two reviewers independently and all disagreements were resolved through discussions.

2.5. Data Extraction and Analysis. The two researchers employed unified data extraction forms and independently extracted data, which included general characteristics of the patient, diagnostic criteria, interventions, follow-up, and efficacy evaluation indicators.

RevMan 5.2.0 software from the Cochrane Collaboration was used for meta-analysis. Relative risk (RR) was used for count data, mean difference (MD) was used for quantity data, and $95 \%$ confidence interval (CI) was used to ascertain the range of the results. The chi-square test was used for assessing the heterogeneity of all clinical trials. For the test of heterogeneity $\left(I^{2}>50 \%, p<0.1\right)$, random effects model (REM) was used to analyze the expression effect, whereas a fixed effects model (FEM) was used to merge data.

\section{Results}

3.1. Process for Including Literatures. Thirty-nine papers were included in this study [1-39]. The literature searching process is shown in Figure 1.

3.2. Characteristics of the Included Studies. 5442 endometriosis patients mentioned in the 39 papers were included in this study, with an average sample size of 140 cases. All papers were published Chinese literatures in mainland China. Dan'e-fukang soft extract alone was used in the 39 papers. The control groups were danazol, gestrinone, mifepristone, and marvelon. Laparoscopic postoperative medication was used in 9 papers $[8,13,17,25,26,31,39-$ 41]. The features of the included literatures are shown in Table 1 .

3.3. The Methodological Quality of Included Studies. The studies were biased with high risks and the quality was low in all included papers. Wherein six papers used a random number table to randomize the groups [9, 28, 34, 39, 42, 43], other papers merely mentioned the word "randomized" in the text. Random allocation concealment and blinding were not mentioned in all the papers. Placebo was used in one paper, but no detailed placebo information was reported [34]. No exit and lost cases were reported in the six papers that were rated as low risk of bias [8, 16, 26, 28, 34, 43]. Exit and lost cases were reported in two papers, but intention analysis was not done; therefore, it was rated as high risk of bias $[6,38]$. No details were reported in other papers, and they were rated as "unclear." Since program registrations were conducted in none of the included studies, the selection bias was "unclear" for most of the studies. However, there were discrepancies in the methodology and results in four papers, and they were rated as high risk of bias $[6,11,16,44]$. The principle for estimating the sample amount was not reported in any of the included studies. The methodological quality of the included studies is shown in Figures 2 and 3. 


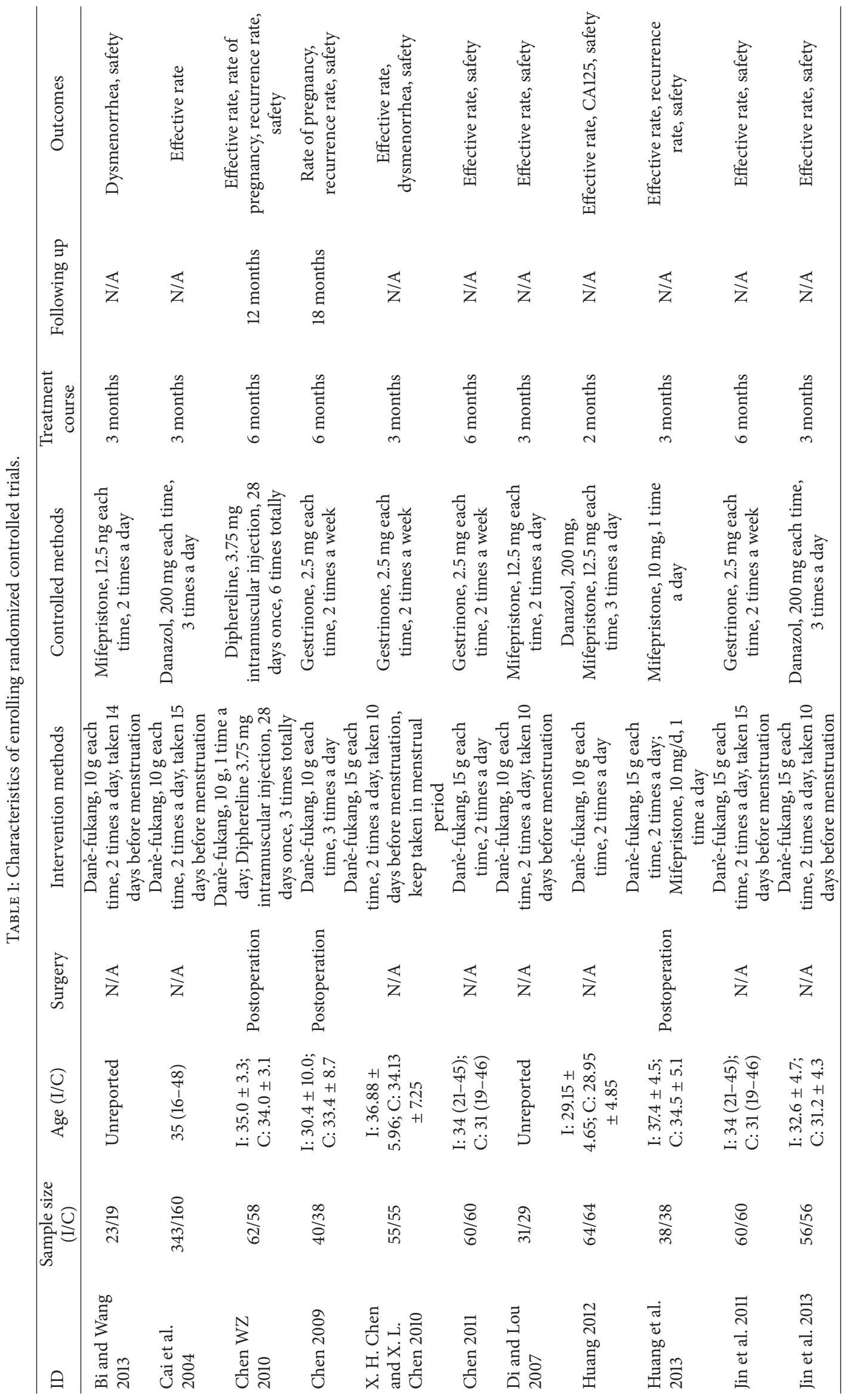




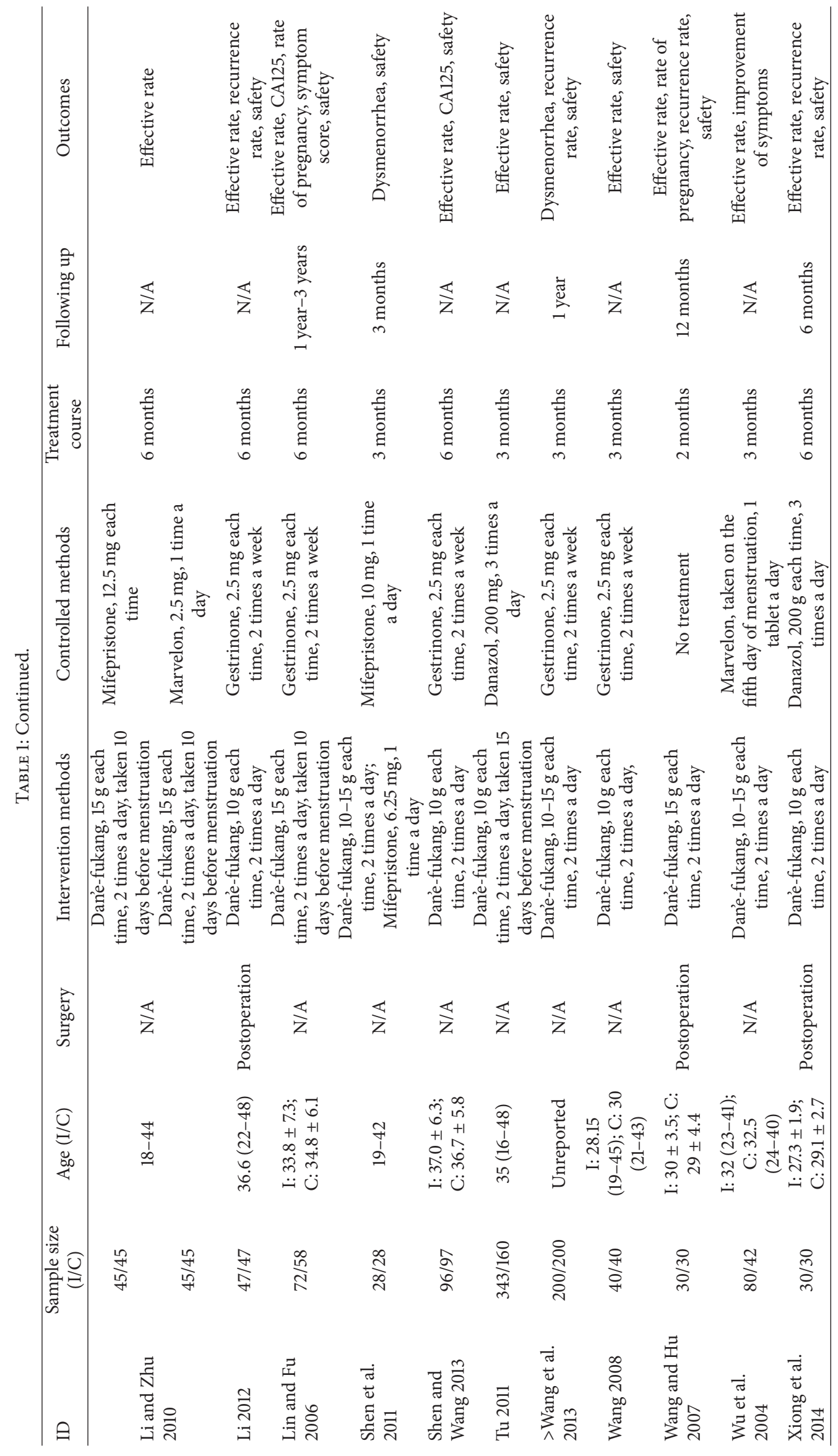




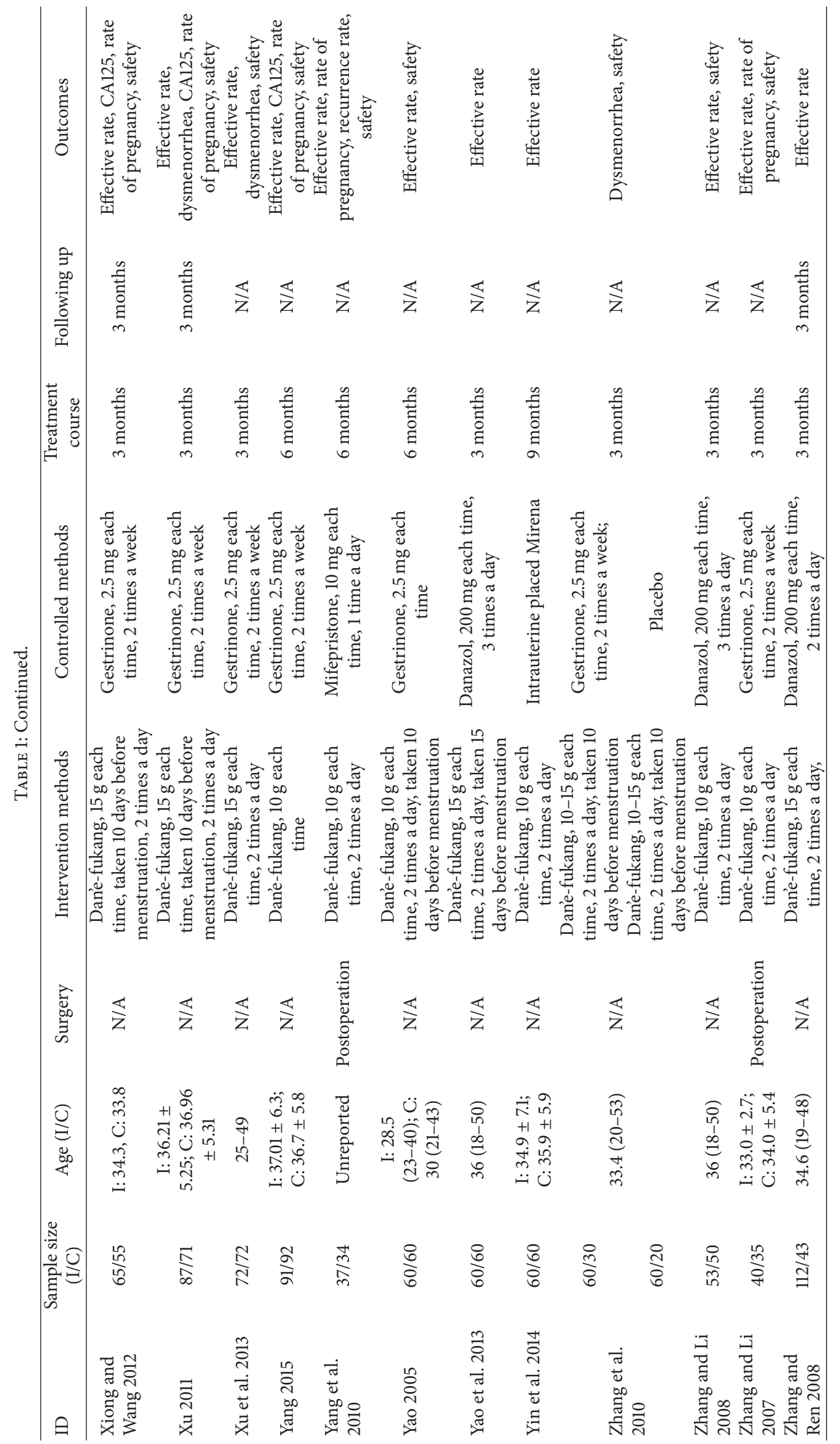




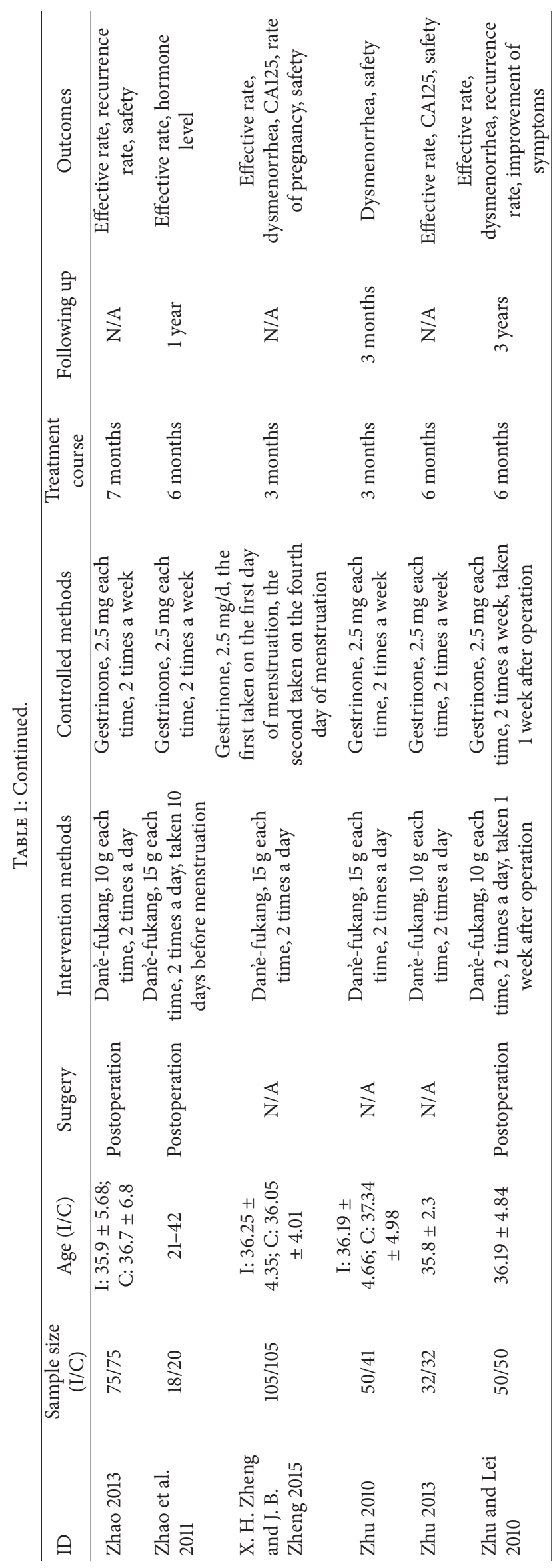




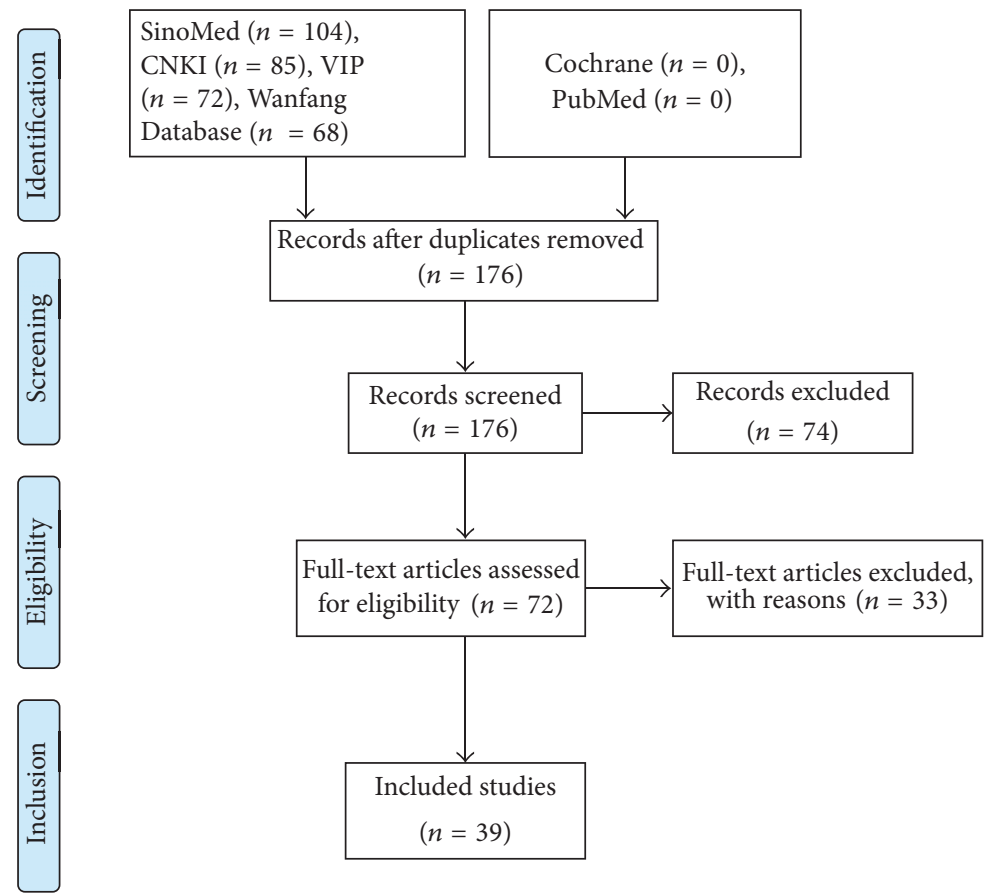

FiguRE 1: PRISMA 2009 flow diagram.

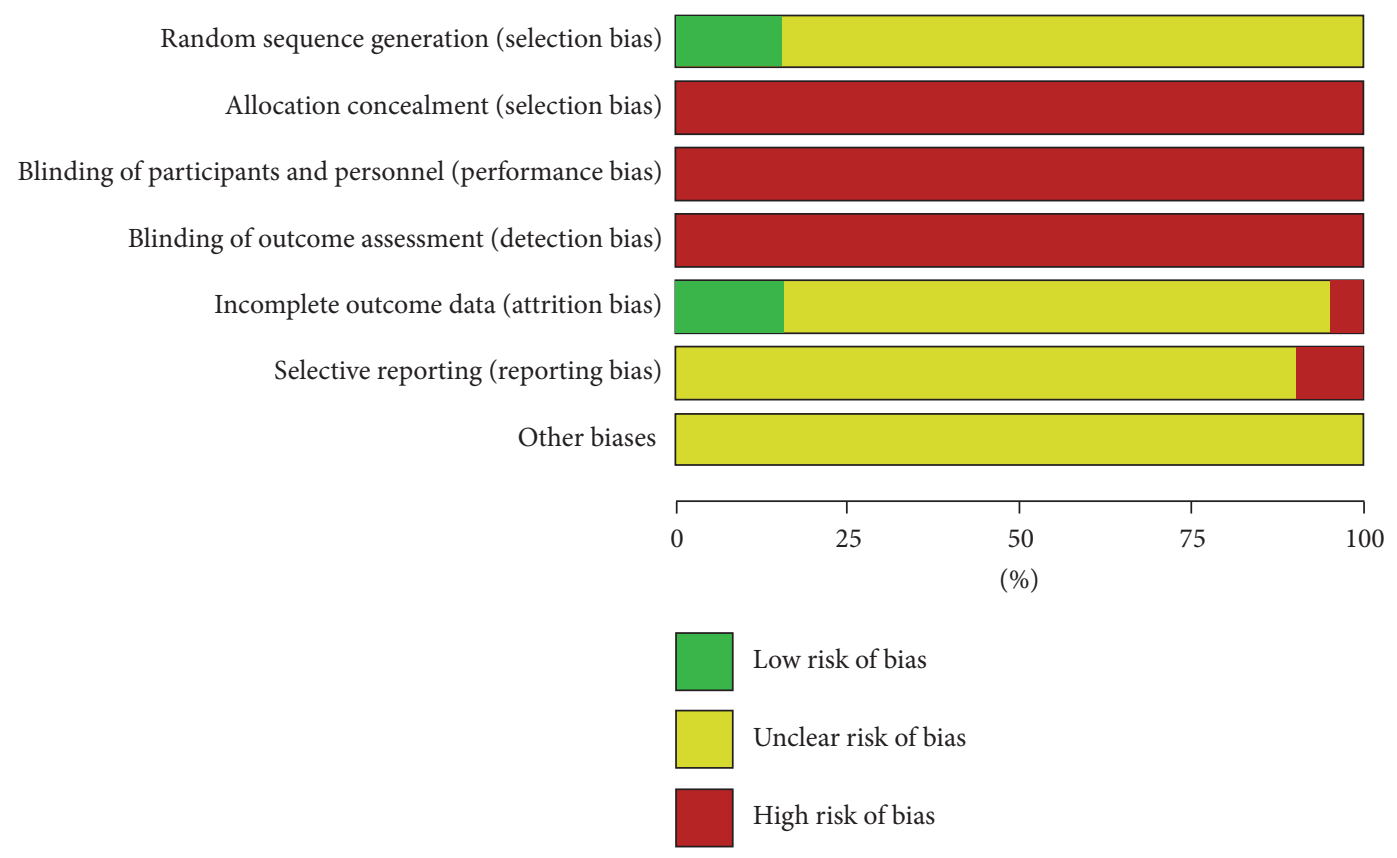

FIGURE 2: Risk of bias graph.

\subsection{Efficiency of Dan'e-Fukang Soft Extract in the Treatment of Endometriosis}

3.4.1. Efficiency. The efficiency of Dan'e-fukang soft extract and gestrinone in the treatment of endometriosis was compared in 18 papers, and there was no significant difference in the efficacy $(P<0.05)$ in 10 papers. However, metaanalysis showed that Dan'e-fukang soft extract was superior to gestrinone $\left(\mathrm{RR}=1.08,95 \% \mathrm{CI}\right.$ from 1.03 to $1.15, I^{2}=71 \%$, REM). The efficiency of Dan'e-fukang soft extract and danazol was compared in 8 papers, and the meta-analysis showed no significant difference in efficacy $(\mathrm{RR}=0.99,95 \% \mathrm{CI}$ from 


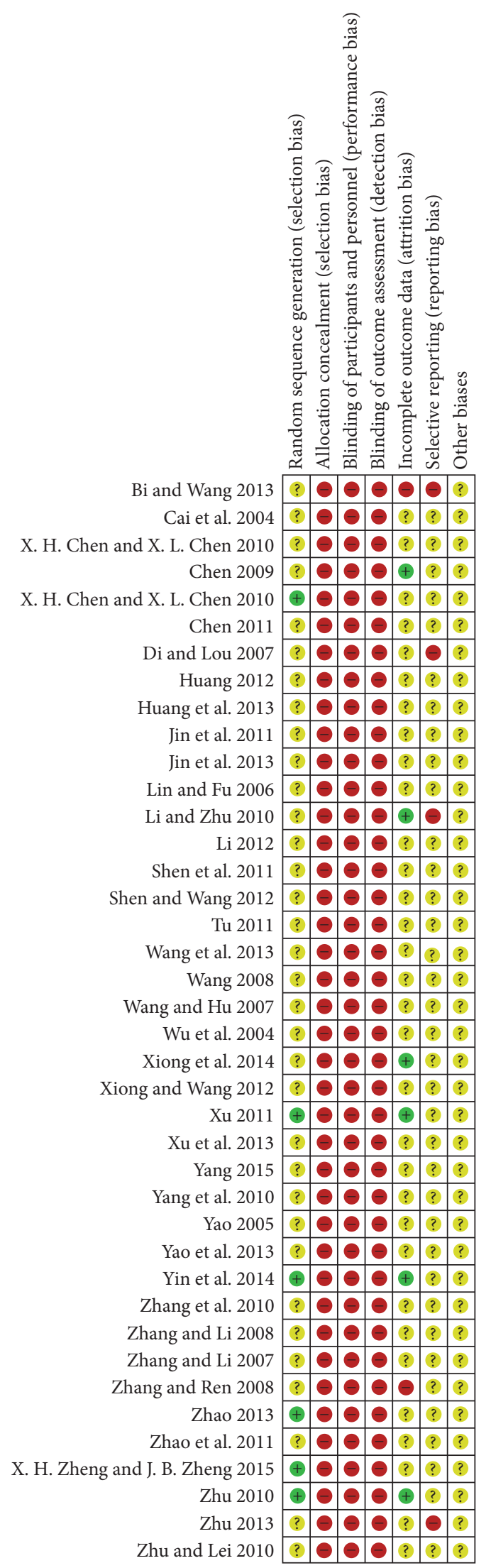

Figure 3: Risk of bias summary. 


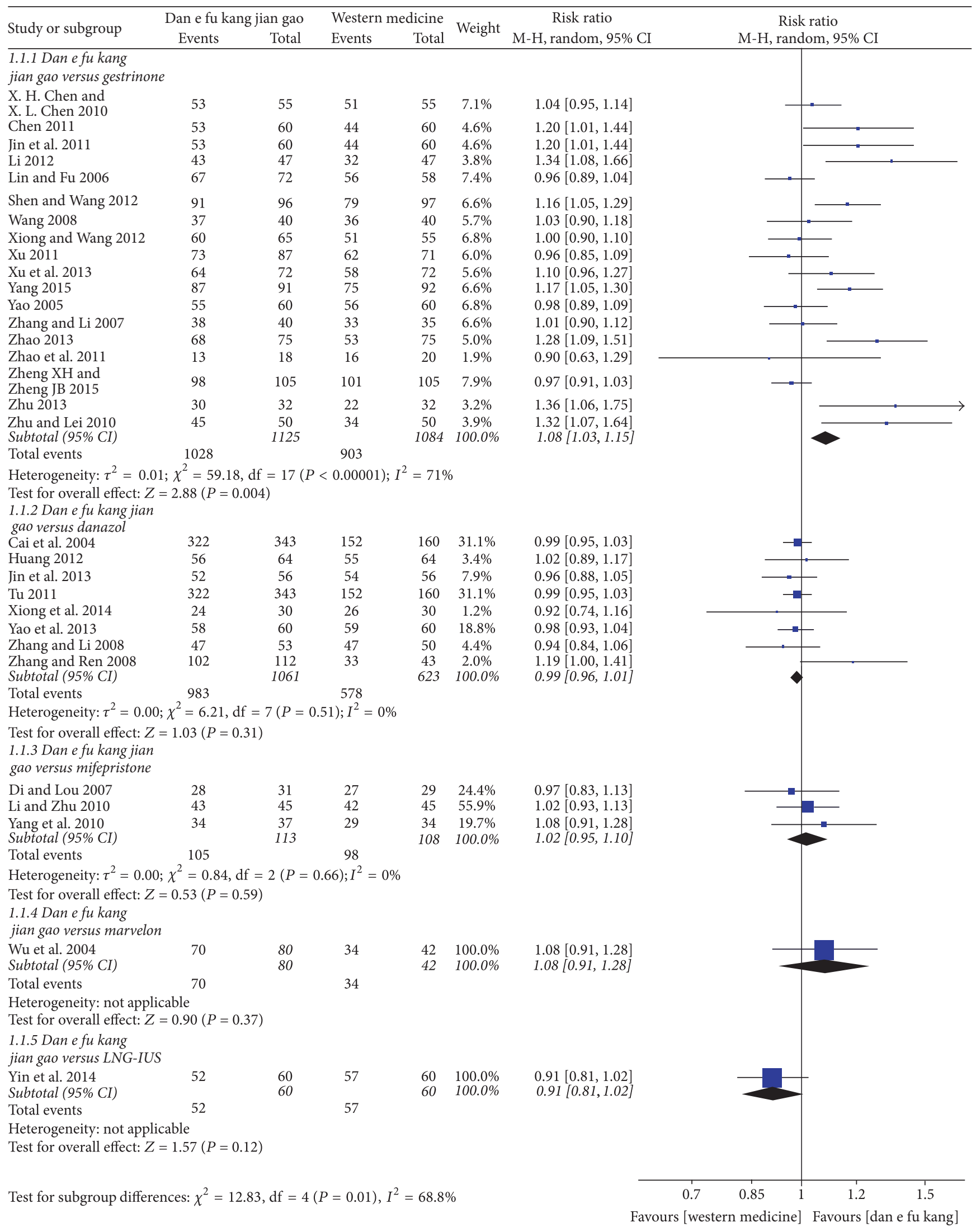

FIGURE 4: Forest plot of effective rate. 


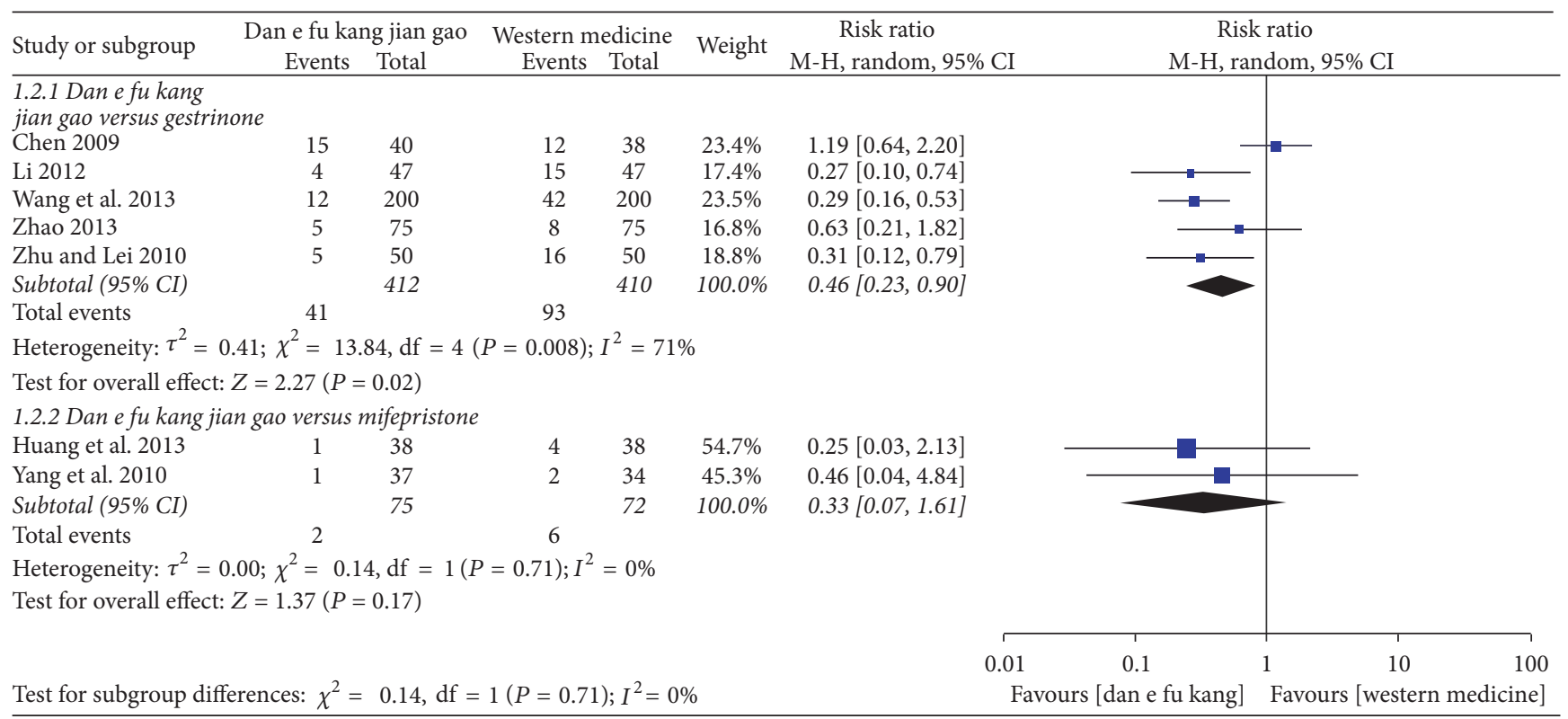

FIGURE 5: Forest plot of recurrence rate.

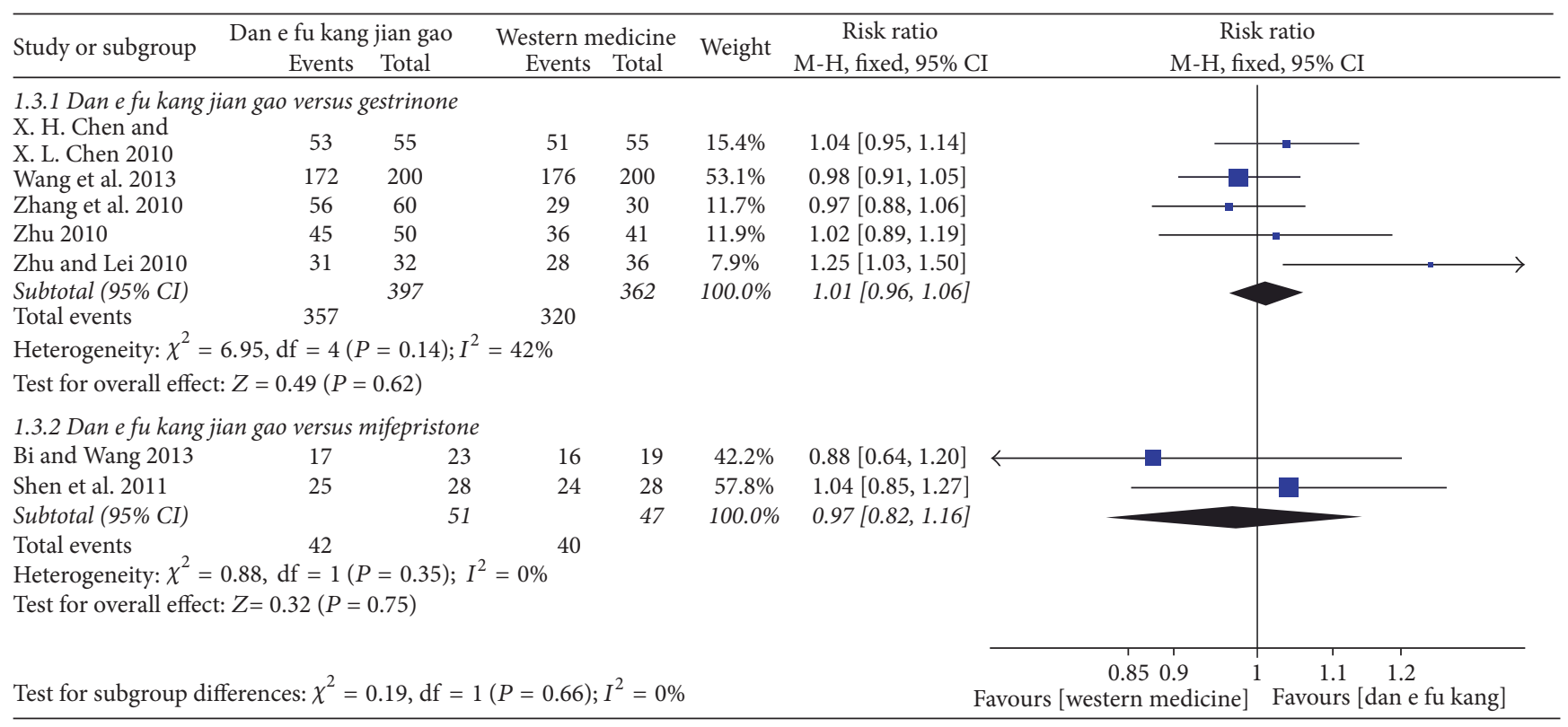

FIgURE 6: Forest plot of dysmenorrhea relieve rate.

0.96 to $1.01, I^{2}=0 \%$, REM). The efficiency of Dan'e-fukang soft extract and mifepristone was compared in 3 studies, and meta-analysis showed no significant difference in efficacy (RR $=1.02,95 \%$ CI from 0.95 to $1.10, I^{2}=0 \%$, REM). The detailed results are shown in Figure 4.

3.4.2. Recurrence Rate. Meta-analysis of five papers showed that the recurrence rate of Dan'e-fukang soft extract was lower than gestrinone in the treatment of endometriosis $(\mathrm{RR}=$ $0.46,95 \%$ CI from 0.23 to $0.90, I^{2}=71 \%$, REM). However, meta-analysis of two papers showed that there was no significant difference between Dan'e-fukang soft extract and mifepristone in reducing the recurrence rate of endometriosis $\left(\mathrm{RR}=0.33\right.$, 95\% CI from 0.07 to $1.61, I^{2}=0 \%$, REM). The detailed results are shown in Figure 5.

3.4.3. Degree of Ease of Dysmenorrhea. The meta-analysis of five papers showed that there was no significant difference between Dan'e-fukang soft extract and gestrinone in the treatment of endometriosis and remission rate of dysmenorrhea ( $\mathrm{RR}=1.01,95 \% \mathrm{CI}$ from 0.96 to $1.06, I^{2}=42 \%$, FEM). The meta-analysis of two papers showed no difference between 


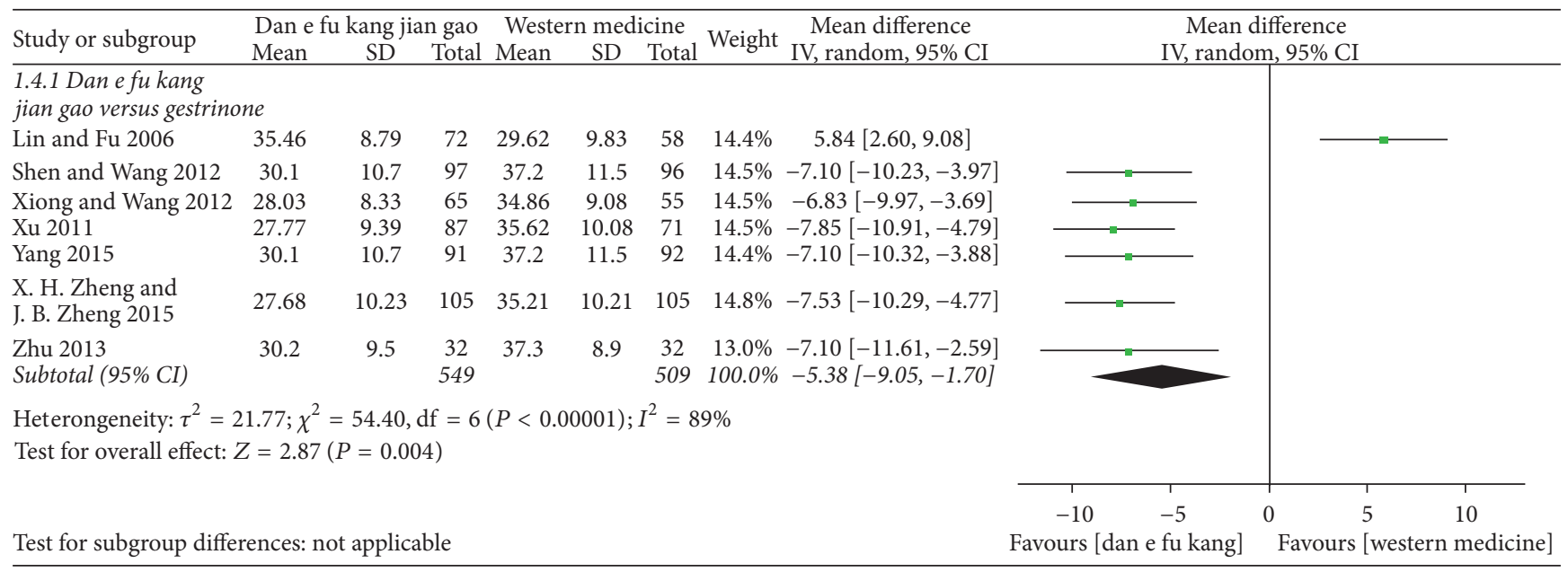

FIGURE 7: Forest plot of CA125.

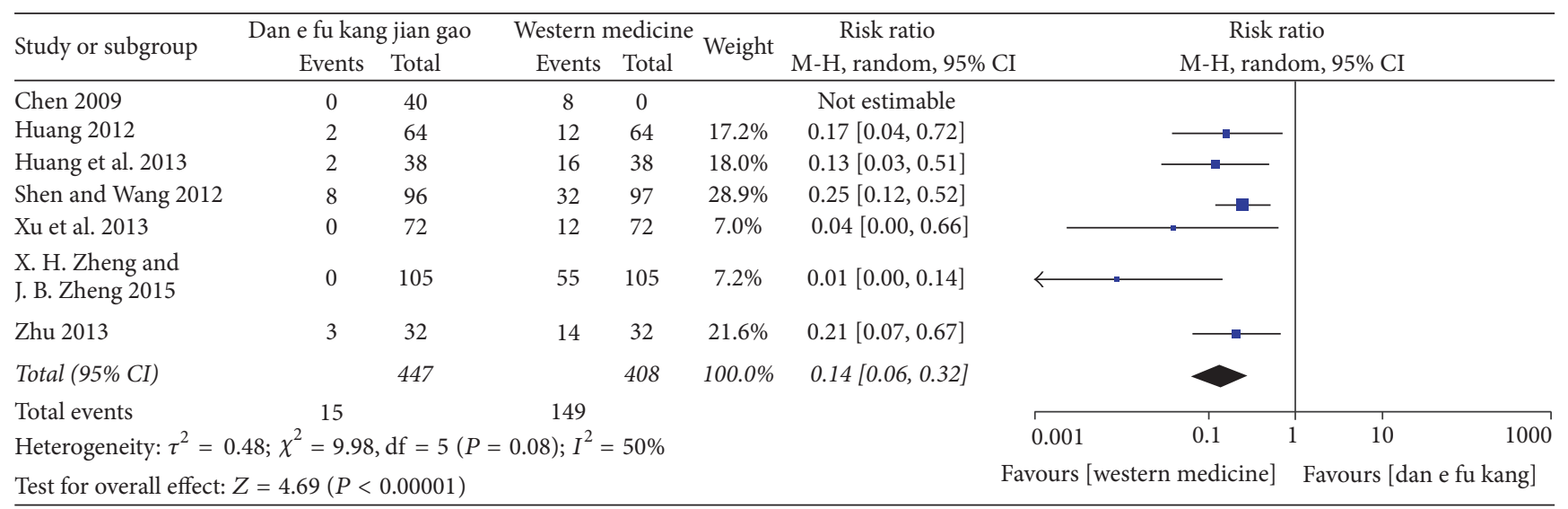

FIGURE 8: Forest plot of adverse reactions.

Dan'e-fukang soft extract and mifepristone in terms of relief of dysmenorrhea ( $\mathrm{RR}=0.97,95 \% \mathrm{CI}$ from 0.82 to $1.16, I^{2}=$ $0 \%$, FEM). The detailed results are shown in Figure 6.

3.4.4. CA125. Meta-analysis of seven papers showed that Dan'e-fukang soft extract was superior to gestrinone in the treatment of endometriosis by regulating CA125 (MD = $-5.38,95 \%$ CI from -9.05 to $-1.70, I^{2}=89 \%$, REM). The detailed results are shown in Figure 7.

3.4.5. Adverse Reactions. No significantly adverse reactions occurred in the Dan'e-fukang soft extract-treated groups in 18 papers, while adverse reactions, such as weight gain, acne, menstrual disorders, abnormal vaginal bleeding, and abnormal liver function, were reported in the western medicinetreated control groups. However, no further meta-analysis could be done because the various symptoms were not standardized. The overall incidence of adverse reactions was reported in six studies and the meta-analysis showed that the adverse reactions in the Dan'e-fukang soft extract group were less than that of the western medicine groups $(\mathrm{RR}=0.14,95 \%$
$\mathrm{CI}=0.06$ to $\left.0.32, I^{2}=50 \%, \mathrm{REM}\right)$. The detailed results are shown in Figure 8.

\section{Discussion}

Endometriosis is a common hormone-dependent gynecological disease, mainly treated with surgeries and western medicines. The recurrence rate is high because invisible lesions cannot be effectively removed with surgeries. The commonly used drugs include gestrinone, danazol, and mifepristone. Not only are they expensive, but also have serious drug side effects, commonly causing damage to the liver and kidney. Moreover, they induce masculinity, which greatly affects the quality of life in patients.

Dan'e-fukang soft extract is composed of two key herbs: Danshen (Salvia miltiorrhiza) and Ezhu (Curcuma zedoaria). Salvia miltiorrhiza, well known for its characters in treating of heart and vascular diseases, has also been explored extensively for treating other diseases [45, 46], and it is documented in the United States Pharmacopeial Convention [47]. Classified by structural characteristics and chemical 
properties, the compounds isolated from Danshen can be categorized as water-soluble and lipid-soluble constituents [48]. Water-soluble constituents mainly exhibit cardiovascular protective activities [49-51]. The lipid-soluble constituents show properties of anticancer and anti-inflammation [5255]. Family Zingiberaceae consisting of about 1400 species and 47 genera has been used in medicine for centuries [56]. Ezhu (Curcuma zedoaria) also known as white turmeric, kachur, and zedoary is a continuing herb belonging to family Zingiberaceae which is cultivated all over Asia. It is used traditionally to treat inflammation, pain, and a variety of skin ailments including wounds, as well as menstrual irregularities and ulcers [57]. Curcuma zedoaria is being used as antiinflammatory, carminative, antitumor, gastrointestinal stimulant, antiulcer, stomachic, antiallergic, diuretic, hepatoprotective, antinociceptive, demulcent, expectorant, rubefacient, and antimicrobial agents [57-61]. Endometriosis, based on an estrogen-inflammation dependent and blood supply disorder [62], Danshen (Salvia miltiorrhiza), and Ezhu (Curcuma zedoaria), theoretically, are better choices for the treatment on the disease.

The results of this study showed that Dan'e-fukang soft extract was superior to gestrinone in treatment of endometriosis, and its efficiency was comparable to that of danazol and mifepristone. It is important to note that adverse reactions in Dan'e-fukang soft extract group were significantly lower than the western medicine group, indicating that Dan'e-fukang soft extract can improve endometriosis to a certain degree, and it is worth further exploration. However, the quality of the literature included in this study was generally low; therefore, a firm conclusion could not be drawn.

The generally low methodological quality of the included literature is a limitation of this study. The reasons for the low quality are as follows: (1) some subjective bias may exist as no placebo-controlled and blinded trials were implemented in the studies; (2) selective reporting bias cannot be ruled out as no proposals were registered and published in the studies; and (3) no detailed randomization methods were reported in most studies and the term "randomized" was merely mentioned. Therefore, only a part of the studies is true "randomized controlled trials"; (4) the efficacy evaluation of the included studies was mainly based on compound outcomes; for example, the degree of improvement based on multiple symptoms is divided into four levels: cured, obvious effective, effective, and ineffective. Since judging criteria in the studies are inconsistent, misclassification bias might exist. In future, rigorously designed, large-scale, multicenter RCTs are recommended to further validate the efficacy of Dan'efukang soft extract and to draw more reliable conclusions.

\section{Conclusion}

The results of this study show that Dan'e-fukang soft extract offers certain advantages in endometriosis treatment. However, because the methodological quality of the included studies was low, rigorously designed and strictly implemented RCTs are needed to further validate its efficacy.

\section{Competing Interests}

The authors declare that there is no conflict of interests regarding the publication of this article.

\section{Acknowledgments}

The authors thank Ms. Yalin Song for assistance with data extraction.

\section{References}

[1] L. C. Giudice, "Endometriosis," New England Journal of Medicine, vol. 362, no. 25, pp. 2389-2398, 2010.

[2] P. Viganò, F. Parazzini, E. Somigliana, and P. Vercellini, "Endometriosis: epidemiology and aetiological factors," Best Practice \& Research Clinical Obstetrics \& Gynaecology, vol. 18, no. 2, pp. 177-200, 2004.

[3] L. H. Wang, "Advances in research on malignant transformation of endometriosis," Progress in Obstetrics and Gynecology, vol. 11, no. 5, p. 369, 2002.

[4] X. J. Liu and Y. L. He, "Establishment and application of endometriosis animal model," Chinese Journal of Practical Gynecology and Obstetrics, vol. 18, no. 7, pp. 444-445, 2002.

[5] J. P. T. Higgins, D. G. Altman, and J. A. C. Sterne, Cochrane Handbook for Systematic Reviews of Interventions Version, Cochrane Collaboration, 2011, http://handbook.cochrane.org/.

[6] X. Y. Bi and D. D. Wang, "Clinical experience of Dan'e-fukang soft extract and mifepristone in the treatment of dysmenorrhea caused by endometriosis," Guide of China Medicine, vol. 11, no. 21, pp. 682-683, 2013.

[7] L. S. Cai, K. Wu, J. P. Yang, and Y. X. Pan, "Observation of the efficacy of Dan'e-fukang extract in the treatment of endometriosis," Chinese Journal of Practical Gynecology and Obstetrics, vol. 20, no. 8, pp. 495-496, 2004.

[8] X. Chen, "Clinical analysis of laparoscopic surgery combined with Chinese and western medicine in the treatment of endometriosis," Journal of Chinese Physician, vol. 11, no. 9, pp. 1251-1252, 2009.

[9] X. H. Chen and X. L. Chen, "Researches on Dan'e-fukang soft extract for chronic pelvic pain caused by endometriosis," Journal of Chinese Physician, vol. 17, no. 30, pp. 24-27, 2010.

[10] Y. Q. Chen, "Observation of clinical efficacy of Dan'e-fukang soft extract in the treatment of endometriosis," Jilin Medicine, vol. 32, no. 2, pp. 467-468, 2011.

[11] W. Di and W. H. Lou, "Clinical observation of Dan'e-fukang soft extract in treatment of endometriosis," Journal of China-Japan Friendship Hospital, vol. 21, no. 5, pp. 314-315, 2007.

[12] L. M. Huang, "Clinical observation on 64 cases of endometriosis treated with Dan'e-fukang soft extract," Hainan Medical Journal, vol. 23, no. 10, pp. 73-74, 2012.

[13] X. F. Huang, H. M. Yang, and Q. M. Chen, "Clinical efficacy of mifepristone combined with Dan'e-fukang soft extract in the treatment of endometriosis," Chinese Journal of Clinical Rational Drug Use, vol. 6, no. 9, pp. 67-68, 2013.

[14] L. Y. Jin, Y. Lu, and Y. L. Shang, "Observation of clinical efficacy of Dan'e-fukang soft extract in the treatment of endometriosis," Journal of Shandong University of Traditional Chinese Medicine, vol. 35, no. 2, pp. 138-139, 2011. 
[15] X. Y. Jin, F. Y. Meng, and Z. Z. Luo, “64 Cases of Endometriosis treated with Dane-fukang soft extract," China Pharmaceuticals, vol. 22, no. 4, pp. 89-90, 2013.

[16] Y. M. Li and X. Q. Zhu, "Clinical study of endometriosis treated with Dan'e-fukang soft extract," Journal of New Medicine, vol. 20, no. 5, pp. 456-458, 2010.

[17] Z. Y. Li, "94 cases of ovarian chocolate cyst with the treatment of post-laparoscopic surgery combined with Dan'e-fukang soft extract," Chinese Medicine Modern Distance Education of China, vol. 10, no. 2, p. 55, 2012.

[18] X. Lin and L. H. Fu, "Clinical observation of Dan'e-fukang soft extract in the treatment of endometriosis," Guangxi Journal of Traditional Chinese Medicine, vol. 29, no. 3, pp. 10-11, 2006.

[19] H. C. Shen, X. J. Zhang, Y. Q. Wang, and S. Y. Wu, "Clinical observation of Dan'e-fukang soft extract in the treatment of dysmenorrhea induced by endometriosis," Proceeding of Clinical Medicine, vol. 20, no. 11, pp. 829-831, 2011.

[20] X. H. Shen and B. D. Wang, "Efficacy analysis of Dan'e-fukang soft extract in the treatment of endometriosis," China Modern Medicine, vol. 19, no. 9, pp. 107-108, 2012.

[21] Y. Q. Tu, "Efficacy observation of Dan'e-fukang soft extract in the treatment of endometriosis," China Practical Medicine, vol. 6, no. 7, pp. 156-157, 2011.

[22] H. Wang, J. F. Sun, and L. L. Zhao, "Efficacy observation of Dan'e-fukang soft extract in the treatment of dysmenorrhea induced by endometriosis," Journal of Henan Medical College for Staff and Workers, vol. 25, no. 6, pp. 691-692, 2013.

[23] W. R. Wang, "Clinical observation of Dan'e-fukang soft extract in the treatment of endometriosis," Journal of Liaoning University of Traditional Chinese Medicine, vol. 10, no. 1, article 98, 2008.

[24] Y. P. Wang and M. X. Hu, "Efficacy analysis of laparoscopic surgery combined with Dane-fukang soft extract in the treatment of endometriosis," Hubei Journal of Traditional Chinese Medicine, vol. 29, no. 10, p. 39, 2007.

[25] L. L. Wu, J. L. Wang, and F. Tang, "Chinese traditional medicine Dan'e-fukang extract in the treatment of endometriosis," Guangdong Medical Journal, vol. 25, no. 6, pp. 728-729, 2004.

[26] H. L. Xiong, Z. Y. Zhao, and Y. P. Jiang, "Efficacy analysis of laparoscopic surgery combined with Dan'e-fukang soft extract in the treatment of endometriosis," Laboratory Medicine and Clinic, vol. 11, no. 17, pp. 2359-2361, 2014.

[27] X. Xiong and Z. H. Wang, "65 Cases of endometriosis with the treatment of Dan'e-fukang soft extract," Fujian Medical Journal, vol. 34, no. 6, pp. 68-70, 2012.

[28] H. Xu, "Efficacy and safety of Dan'e-fukang soft extract in the treatment of endometriosis," Capital Medicine, vol. 11, no. 22, pp. 34-35, 2011.

[29] Z. X. Xu, H. J. Ma, D. L. Fang, and L. Q. Zhou, "Analysis of the clinical efficacy and safety of Dan'e-fukang soft extract in the treatment of endometriosis," Maternal and Child Health Care of China, vol. 28, no. 12, pp. 1880-1881, 2013.

[30] J. Y. Yang, "Efficacy Analysis of Dan'e-fukang soft extract in patients with endometriosis," Anti-Infection Pharmacy, vol. 12, no. 3, pp. 452-453, 2015.

[31] L. F. Yang, C. L. Zhu, and Q. F. Xu, "Clinical observation of Dan'e-fukang soft extract and mifepristone adjuvant therapy for postsurgical endometriosis," Chinese Medical Innovations, vol. 7, no. 10, pp. 52-53, 2010.

[32] J. S. Yao, "Clinical comparative observation between Chinese Traditional Medicine and Nemestran in the treatment of endometriosis," Journal of Jinzhou Medical College, vol. 26, no. 4, p. 88, 2005.

[33] X. Y. Yao, ZH, D. Bao, and X. L. Liu, "Efficacy of Dan'e-fukang soft extract in the treatment of endometriosis and its effect on patients' anxiety and depression," Chinese Journal of Obstetrics \& Gynecology and Pediatrics, vol. 9, no. 1, pp. 97-98, 2013.

[34] Y. Y. Yin, N. Li, and J. Y. Pan, "Clinical observation on 60 cases of endometriosis treated with Dan'e-fukang soft extract combined with Mirena," Guiding Journal of Traditional Chinese Medicine and Pharmacy, vol. 20, no. 11, pp. 74-75, 2014.

[35] B. Zhang, J. H. Zhao, and T. Y. Shang, "Efficacy observation of Dan'e-fukang soft extract in the treatment of pelvic pain caused by endometriosis," Proceeding of Clinical Medicine, vol. 19, no. 3, pp. 360-362, 2010.

[36] C. E. Zhang and J. Y. Li, "Efficacy observation of Dan'efukang soft extract in the treatment of endometriosis," Lishizhen Medicine and Materia Medica Research, vol. 19, no. 6, pp. 14781479, 2008.

[37] C. X. Zhang and L. Li, "Efficacy observation on 40 cases of post cystic surgical ovarian endometriosis with adjuvant treatment of Dan'e-fukang soft extract," Chinese Journal of Integrated Traditional and Western Medicine, vol. 27, no. 1, pp. 87-88, 2007.

[38] X. Zhang and Y. L. Ren, "Efficacy observation of Dan'e-fukang soft extract in the treatment of endometriosis," Journal of Medical Forum, vol. 29, no. 7, pp. 48-49, 2008.

[39] G. Y. Zhao, "Clinical efficacy of laparoscopic surgery in combination with drug treatment of ovarian chocolate cyst," Seek Medical, vol. 11, no. 2, article 293, 2013.

[40] Q. L. Zhao, Q. Chen, and J. S. Zhu, "Clinical efficacy observation of Gestrinone and Dan'e-fukang extract in the treatment of severe post-surgical endometriosis," Journal of Lanzhou University (Medical Sciences), vol. 37, no. 4, pp. 54-56, 2011.

[41] Y. Z. Zhu and L. H. Lei, "Clinical efficacy observation of laparoscopic surgery combined with medication in the treatment of ovarian chocolate cysts," Modern Chinese Doctor, vol. 48, no. 35, pp. 20-21, 2010.

[42] X. H. Zheng and J. B. Zheng, "Efficacy observation of Dan'efukang extract in the treatment of Endometriosis," Modern Journal of Integrated Traditional Chinese and Western Medicine, vol. 24, no. 36, pp. 4056-4058, 2015.

[43] F. J. Zhu, "Dan'e-fukang soft extract in the treatment of painful symptoms of endometriosis," Strait Pharmaceutical Journal, vol. 22, no. 4, pp. 104-105, 2010.

[44] W. M. Zhu, “32 Cases of endometriosis treated Dan'e-fukang soft extract," Chinese Medicine Modern Distance Education of China, vol. 11, no. 24, p. 26, 2013.

[45] Y.-B. Wu, Z.-Y. Ni, Q.-W. Shi et al., "Constituents from salvia species and their biological activities," Chemical Reviews, vol. 112, no. 11, pp. 5967-6026, 2012.

[46] F. Long, H. Yang, Y. Xu, H. Hao, and P. Li, "A strategy for the identification of combinatorial bioactive compounds contributing to the holistic effect of herbal medicines," Scientific Reports, vol. 5, Article ID 12361, 2015.

[47] United States Pharmacopoeia Salvia 1-3 Dietary Supplements, USP 37/NF, U. S. Pharmacopoeial Convention, Rockville, Md, USA, 32nd edition, 2014.

[48] X. Chen, J. Guo, J. Bao, J. Lu, and Y. Wang, "The anticancer properties of salvia miltiorrhiza bunge (Danshen): a systematic review," Medicinal Research Reviews, vol. 34, no. 4, pp. 768-794, 2014. 
[49] W. Zeng, W. Shan, L. Gao et al., "Inhibition of HMGB1 release via salvianolic acid B-mediated SIRT1 up-regulation protects rats against non-alcoholic fatty liver disease," Scientific Reports, vol. 5, Article ID 16013, 2015.

[50] H. Fan, M. Yang, D. Qi et al., "Salvianolic acid A as a multifunctional agent ameliorates doxorubicin-induced nephropathy in rats," Scientific Reports, vol. 5, Article ID 12273, 2015.

[51] B. Liu, Y. Du, L. Cong, X. Jia, and G. Yang, "Danshen (Salvia miltiorrhiza) compounds improve the biochemical indices of the patients with coronary heart disease," Evidence-Based Complementary and Alternative Medicine, vol. 2016, Article ID 9781715, 9 pages, 2016.

[52] M. Xu, F. Cao, Y. Zhang et al., "Tanshinone IIA therapeutically reduces LPS-induced acute lung injury by inhibiting inflammation and apoptosis in mice," Acta Pharmacologica Sinica, vol. 36, no. 2, pp. 179-187, 2015.

[53] A. Singh, V. Singh, R. L. Tiwari et al., "The IRAK-ERKp67phox-Nox-2 axis mediates TLR4, 2-induced ROS production for IL-1 $\beta$ transcription and processing in monocytes," Cellular and Molecular Immunology, vol. 13, no. 6, pp. 745-763, 2015.

[54] V. G. D’Agostino, P. Lal, B. Mantelli et al., "DihydrotanshinoneI interferes with the RNA-binding activity of HuR affecting its post-transcriptional function," Scientific Reports, vol. 5, Article ID 16478, 2015.

[55] Y. Yu, S. Hu, G. Li et al., "Comparative effectiveness of Diao Xin Xue Kang capsule and compound danshen tablet in patients with symptomatic chronic stable angina," Scientific Reports, vol. 4, article 7058, 2014.

[56] K. N. Babu, M. Sabu, K. N. Shiva, M. Divakaran, and P. N. Ravindran, "Ginger," in Genetic Resources, Chromosome Engineering, and Crop Improvement: Medicinal Plants, R. J. Singh, Ed., pp. 393-450, CRC Press, Taylor \& Francis Group, Boca Raton, Fla, USA, 2011.

[57] H. M. A. Ullah, S. Zaman, F. Juhara et al., "Evaluation of antinociceptive, in-vivo \& in-vitro anti-inflammatory activity of ethanolic extract of Curcuma zedoaria rhizome," $B M C$ Complementary and Alternative Medicine, vol. 14, no. 1, article no. 346, 2014.

[58] B. Wilson, G. Abraham, V. S. Manju et al., "Antimicrobial activity of Curcuma zedoaria and Curcuma malabarica tubers," Journal of Ethnopharmacology, vol. 99, no. 1, pp. 147-151, 2005.

[59] H. Makabe, N. Maru, A. Kuwabara, T. Kamo, and M. Hirota, "Anti-inflammatory sesquiterpenes from Curcuma zedoaria," Natural Product Research, vol. 20, no. 7, pp. 680-685, 2006.

[60] R. Lobo, K. S. Prabhu, A. Shirwaikar, and A. Shirwaikar, "Curcuma zedoaria Rosc, (white turmeric): a review of its chemical, pharmacological and ethnomedicinal properties," Journal of Pharmacy and Pharmacology, vol. 61, no. 1, pp. 13-21, 2009.

[61] X.-F. Gao, Q.-L. Li, H.-L. Li et al., "Extracts from curcuma zedoaria inhibit proliferation of human breast cancer cell MDA-MB-231 in vitro," Evidence-Based Complementary and Alternative Medicine, vol. 2014, Article ID 730678, 9 pages, 2014.

[62] L. C. Giudice and L. C. Kao, "Endometriosis," Lancet, vol. 364, no. 9447, pp. 1789-1799, 2004. 


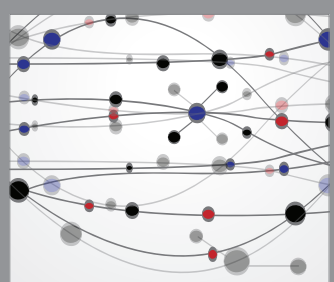

The Scientific World Journal
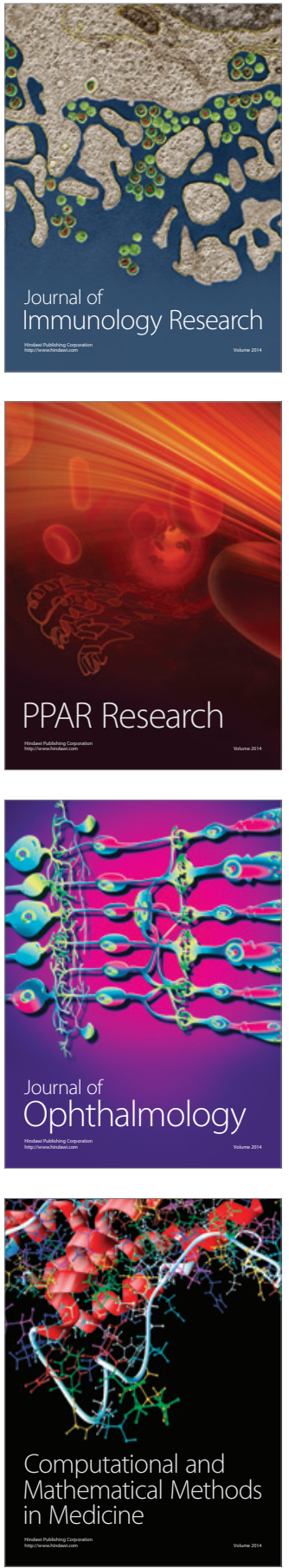

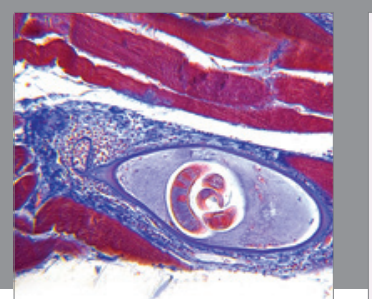

Gastroenterology Research and Practice
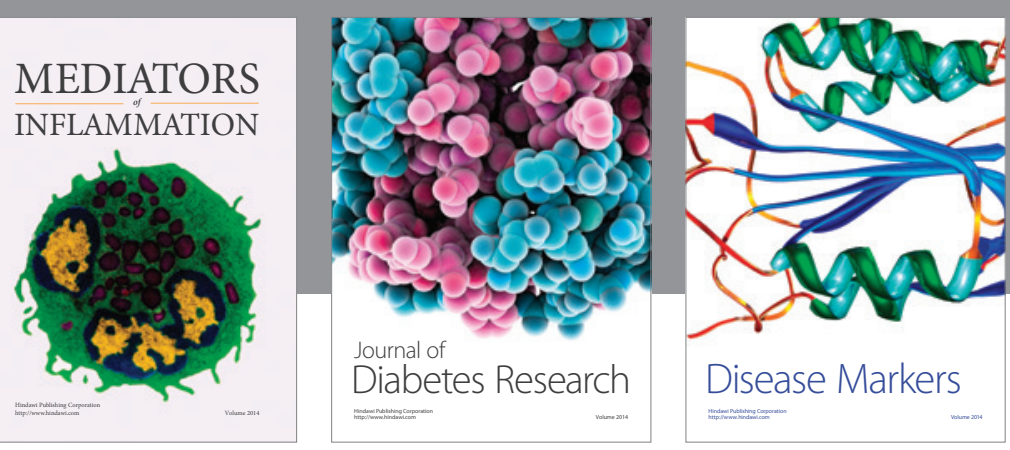

Disease Markers

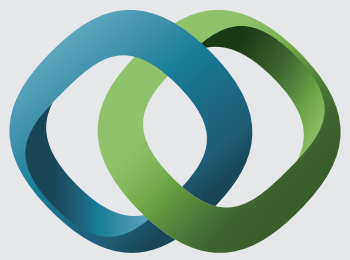

\section{Hindawi}

Submit your manuscripts at

https://www.hindawi.com
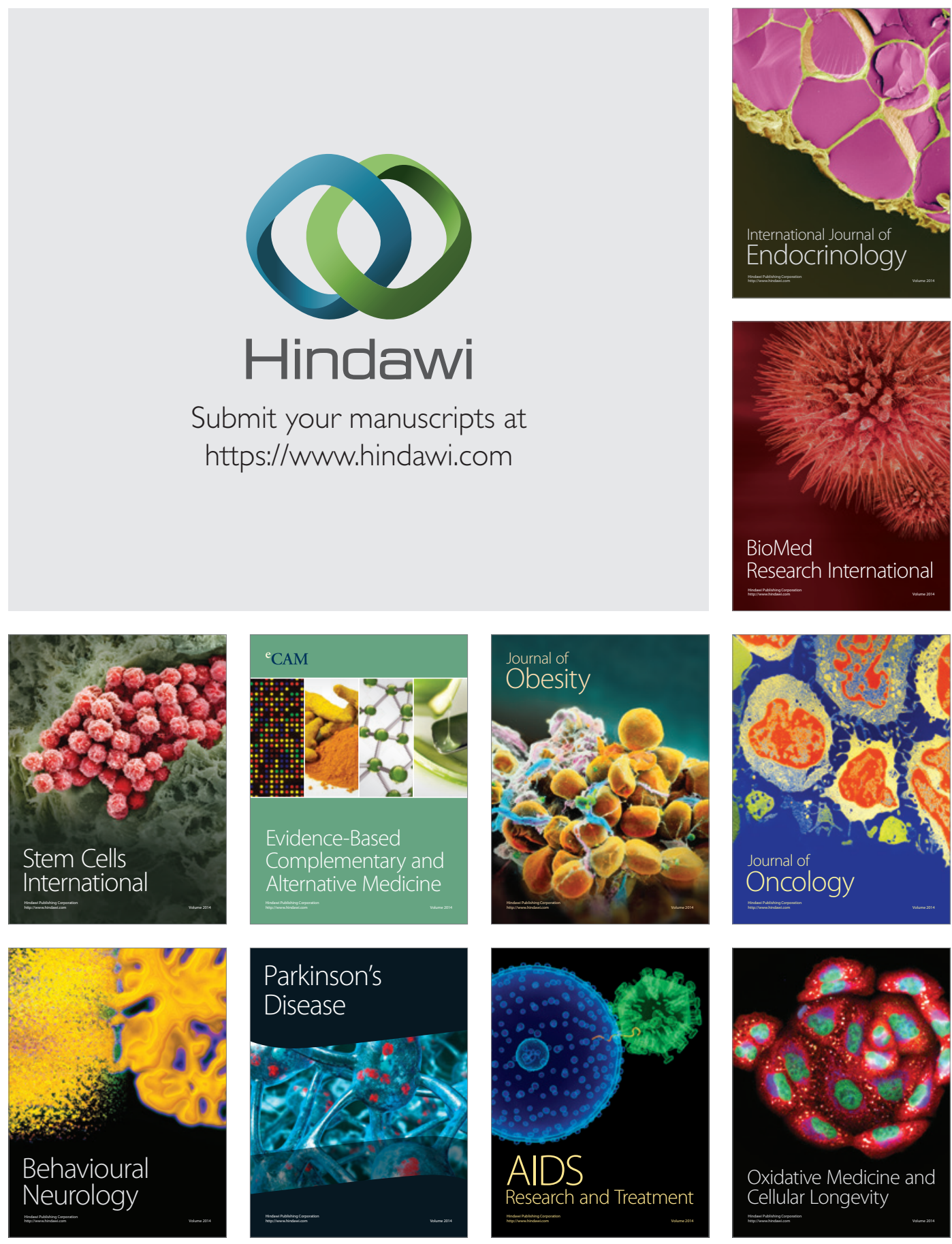